http://jmscr.igmpublication.org/home/ ISSN (e)-2347-176x ISSN (p) 2455-0450 crossref DOI: https://dx.doi.org/10.18535/jmscr/v8i2.38

\author{
Dournal Of Medical Science And Clinical Research \\ IGM Publication \\ An official Publication of IGM Publication
}

\title{
Neonatal Adrenal Hemorrhage Presenting As Late Onset Adrenal Insufficiency: A Case Report
}

Authors

\author{
Dr Shantisena Mishra ${ }^{1}$, Dr Aparna B Raj ${ }^{2 *}$, Dr Sarada Prasanna Pradhan \\ ${ }^{1}$ Associate Professor, Dept. of Pediatrics, MKCG Medical College, Berhampur, Odisha \\ ${ }^{2,3}$ Junior Resident, Dept of Pediatrics, MKCG Medical College, Berhampur, Odisha \\ *Corresponding Author
}

Dr Aparna B Raj

Junior Resident, Dept of Pediatrics, New PG ladies' Hostel, MKCG Medical College, Berhampur, Odisha

Abstract
Neonatal adrenal hemorrhage is a relatively uncommon condition, but could prove detrimental if the
symptoms are not recognized early. Clinical manifestations vary greatly depending on the rate and severity
of bleeding and the extent of adrenal tissue involved. We report a case of neonatal adrenal hemorrhage,
possibly due to birth trauma, which was asymptomatic and went unnoticed during the neonatal period and
presented as adrenal insufficiency in the early infantile period.
Keywords: Neonatal adrenal hemorrhage, hypoadrenalism, Shock, adrenal crises.

\section{Introduction}

Neonatal Adrenal Hemorrhage is a relatively uncommon condition with variable and nonspecific presentation that can cause adrenal insufficiency, shock and death if not recognized early and appropriately treated. Here, we report a case of 1.5 month old male child presenting with shock and metabolic abnormalities pertaining to hypoadrenalism, due to Adrenal Hemorrhage and favourable clinical progression with replacement steroids.

\section{Case Report}

45 day old male baby was admitted to ER with chief complaints of non-bilious vomiting and lethargy since D21 of life. The vomiting was nonbilious and followed almost every feed. There wasn't any history of fever, loose stools, bleeding, seizures or Blood Transfusion.
The baby was born as $2^{\text {nd }}$ order child out of non consanguineous marriage to a 35 year old P2L2 mother, with uneventful AN history and delivered by Vaginal delivery with a birth weight of $2.5 \mathrm{~kg}$ and had cried Immediately after birth and with no history of SNCU admission. The baby was under mixed feeding. The elder sibling was a 20year old girl who was doing well. Though the birth spacing was long, the conception was spontaneous and there wasn't any history of any drug intake by the mother. There was no any contact with TB.

On examination, the $1.7 \mathrm{~kg}$ weighing baby was sick, lethargic and was in circulatory shock. The baby was transferred to PICU and the shock correction was done by iv fluids and inotropes, and was started on antibiotics as per the institutional protocol presuming to be a case of Sepsis. RBS was normal, routine blood investigations revealed normocytic normochromic 
anemia with $\mathrm{Hb} 8.8 \mathrm{gm} / \mathrm{dl}$. Serum sodium and potassium levels were deranged (Na-99,118,129 meq/l and $\mathrm{K}$ 6.3,3.9 and 4 meq/L) which got normalized by supportive therapy and sodium correction. In view of multiple episodes of vomiting and abdominal distension, Abdominal Ultrasonogram was done which revealed irregular heterogenous space occupying lesion with central hypoechogenicity in right suprarenal region suggestive of Adrenal hemorrhage, which was again confirmed by a repeat USG and later by CECT Abdomen. (fig1). Sr. Cortisol was found to be low at 2.61 micrograms/dl (Ref Level:6.2-19.4) and Sr. ACTH was found to be high with value $277 \mathrm{pg} / \mathrm{ml}$ (Ref normal <46). After obtaining Endocrinology Consultation, replacement steroids oral Hydrocortisone and Fludrocortisone was started, following which the baby improved symptomatically and showed good increments in daily weight gain and hence discharged to home with advise to serial follow up in OPD.

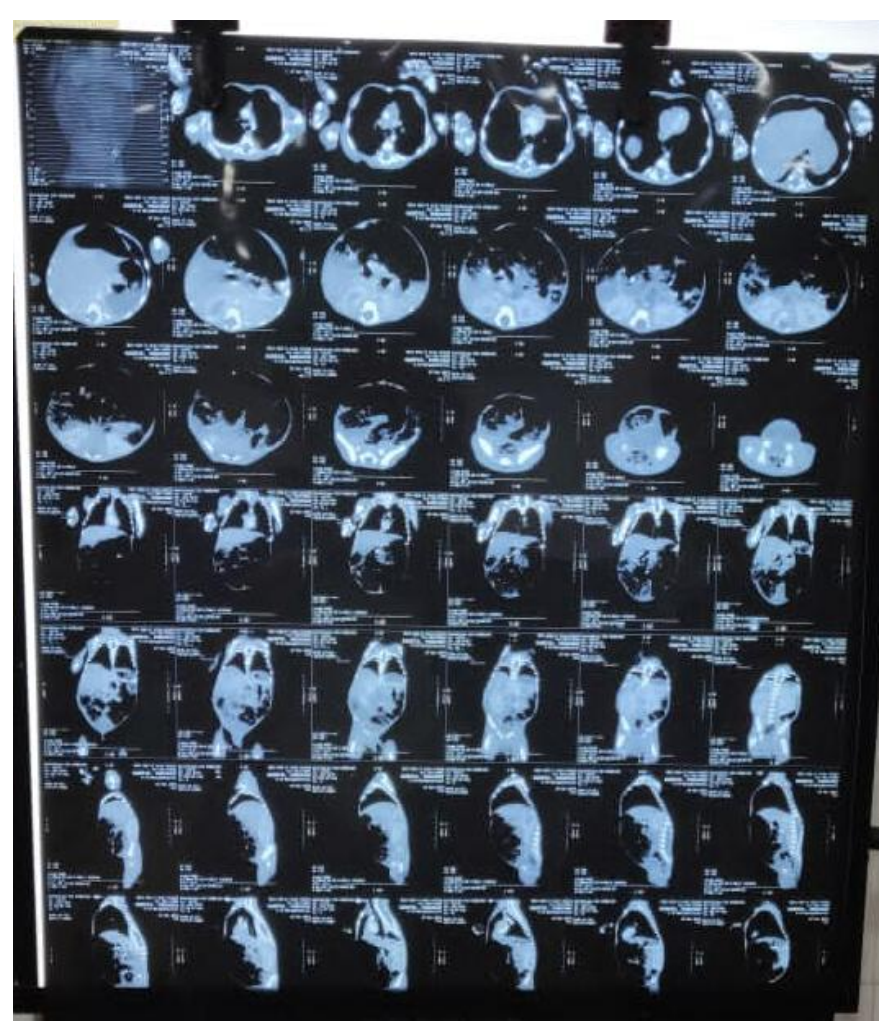

Fig 1 CECT Abdomen showing $23 \times 13 \times 23 \mathrm{~mm}$ size hypodense lesion with minimal peripheral calcific foci and hyperdensity in right suprarenal gland suggestive of adrenal hemorrhage

\section{Discussion}

Neonatal Adrenal Hemorrhage is a relatively uncommon condition with incidence ranging between 0.2 to $0.55 \% .{ }^{1}$ Various risk factors have been reported like birth trauma, birth asphyxia, sepsis, coagulation disorders, perinatal injuries etc. but in a large majority of causes, it can occur without any identifiable cause as well.. In newborns, large size and increased vascularity of adrenal gland may result in vulnerability to mechanical compression and sensitivity to changes in venous pressure during delivery. The increased pressure associated with congestion and damaged endothelial cells associated with hypoxia may cause adrenal hemorrhage. ${ }^{2}$

Neonatal adrenal hemorrhage is usually Unilateral (70\%) and commonly affects Right side gland. ${ }^{3}$ This is because Right adrenal gland blood flow directly drains into IVC and hence is more frequently affected by venous pressure changes and also because it may be easily compressed between liver and the ribs.

NAH is most commonly observed in term babies, that too males for unknown reasons. The presentation is highly variable and non specific in that it may be asymptomatic or may be accompanied by anemia, persistent jaundice, abdominal mass, painful swelling of scrotum or shock. As the gland has good regenerative potential, most NAH are not associated with Adrenal Insufficiency. But, rarely it may cause Adrenal insufficiency too leading to a myriad of metabolic abnormalities like Hyponatremia, Hyperkalemia and the child may present with shock and failure to thrive as was in our case. ${ }^{4}$

The investigation of choice is USG as its easily available and has no harmful radiation exposure. And in the USG, NAH will be usually seen as hypoechoic lesion with calcifications rarely. The Differential Diagnosis includes neuroblastoma, wilm's tumour, Multicystic dysplastic kidney, adrenal cyst, abscess etc. ${ }^{5}$

NAH is usually self limited and complete regression occurs within 5-6 months. ${ }^{6}$ If associated adrenal insufficiency is there, steroids are to 
replaced and serial follow up has to be done with USG and Sr. electrolytes and Sr. ACTH, Sr. cortisol. The associated Adrenal insufficiency is usually transient, and the steroids can be withdrawn after few months. But the literature review also reports cases where Adrenal insufficiency following $\mathrm{NAH}$ being Permanent and needing long term therapy.

\section{Conclusion}

This case encourage clinicians to keep in mind Adrenal Insufficiency while evaluating an infant for shock in the absence of an infectious etiology and emphasizes Adrenal Hemorrhage as a cause for Adrenal Insufficiency especially in early infantile period.

\section{References}

1. Bergami G, Malena S, Di Mario M, Fariello G. Ecography in the follow up of Neonatal Adrenal Hemorrhage. The presentation of 14 cases. Radiol Med. 1990 ; 79(5):474-8.

2. Abdu AT, Kriss VM, Bada HS, Reynolds EW. Adrenal Hemorrhage in a newborn. Am J Perinatal. 2009: 26;553-557.

3. Singh J, Dalal P, Bhalla K, Rattan KN. Neonatal Adrenal Hemorrhage presenting as prolonged NNH. J Neonatal Surg.2016; 5(2):22

4. Eklof O, Grotte G, Jorulf H, Lohr G, Ringertz H. Perinatal Hemorrhagic necrosis of the adrenal gland. A clinical and radiological evaluation of 24 consecutive cases. Pediat Radiol. 1975:4:31-36.

5. Velaphi SC, Perlman JM. Neonatal adrenal hemorrhage: clinical and abdominal sonographic findings. Clinical Pediatrics 2001.40 545-548. (Pub Med)

6. Postek G, Streich H, Narebskisk Assessment of diagnostic methods in adrenal gland hemorrhage in newborns on the basis of own material from the years 2007-2011. Pol J Radiol.2011 : 76(3) : 62-4. 